\title{
$\sqrt{-1} \frac{\text { EasyChair Preprint }}{\text { № } 517}$
}

\section{A Blockchain Aided Metric for Predictive Delivery Performance in Supply Chain Management}

Mark H. Meng and Yaou Qian 


\section{A Blockchain Aided Metric for Predictive Delivery Performance in Supply Chain Management}

\author{
Mark H. Meng \\ National University of Singapore \\ e0001308@u.nus.edu
}

\author{
Yaou Qian \\ Monash University \\ yqia70@student.monash.edu
}

\begin{abstract}
The topic of blockchain has been inundated with the fanaticism of cryptocurrencies' enthusiasts in recent years. In fact, the theory of blockchain and technologies behind are more worthy to be discussed to bring revolution to nowadays business operation. In this paper, we conduct an interdisciplinary study on business logistics as well as the cutting edge information technologies. We discuss the ongoing projects of blockchain in business industry, and then we propose our assessment model and blockchain framework to seek an enhanced metric for delivery performance with real-time feature and higher accuracy. Based on the our analysis, we identify the shortcomings of traditional assessment on delivery performance in contemporary global supply chain management and we discuss the potential benefits brought by adoption of our proposed framework.

Index Terms-blockchain, business intelligence, delivery, smart contract, supply chain management
\end{abstract}

\section{INTRODUCTION}

Blockchain starts drawing people's attention as a cryptocurrency called "Bitcoin" [1]. Blockchain is a novel approach to build trust in a trustless environment and provide integrity, traceability and security to the data management [2], [3]. However, the application of blockchain in early stage only focuses on simple cryptocurrency transactions until the introduction of "smart contract" [4]. Smart contract enables users to apply blockchain technology into a larger extent as it supports more types of operation and offers a programmatic solution to process the data on the ledger. In recent years, many researchers and business organizations from different industries have started putting effort in the study of blockchain and discussing the feasibility of adopting it to reduce operational risk and cost [3]. Some major players in a specific industries like IBM and Maersk have already enrolled the blockchain into their business operation [5].

Supply chain is one of the fields that may benefit most from blockchain technology [6]. In the context of globalization, the diversity and complexity of supply chain composition, great amount of uncertainty in external environment and the shorter life cycle of new product development make supply chain management critical to the profitability or even the survival of a company. Delivery is the very last step to pass the promised goods or services to the customers. Any incident or failure occurs during delivery could certainly result in a regretful customer satisfaction and even possibly lead to negative impact to the future operation of the organization [7]. The difficulty in delivery performance assessment is one of the biggest problems that the industry suffers over the decades.

In this paper, we are going to present an assessment model for predictive delivery performance metric and a blockchain framework designed for industrial supply chain management called DelivChain. By introducing the details of the architecture of DelivChain, we will explain why it could bring benefits to supply chain management from multiple perspectives.

In the following sections, we will first introduce the background of the blockchain technology and its application in industry, especially in supply chain. In Section III, we will present our assessment model and deduction procedure from a standard formula to calculate delivery performance. We then depict the architecture and functionality of DelivChain framework in Section IV. Section V shows our discussion about the benefits of DelivChain that could bring to nowadays supply chain. In the end, we will mention our future plan in Section VI and then we conclude this paper in Section VII.

\section{BACKGROUND}

The topic discussed in this paper is an interdisciplinary study to bring the application of cutting edge technologies to the real business operation. In this section, we present a brief overview on the blockchain technology. Meanwhile we introduce the past and ongoing well known industrial applications of blockchain and thereby discuss the potential advantage of applying blockchain into the supply chain management.

\section{A. Blockchain Technology and Smart Contract}

The nature of blockchain technology is a novel approach for data management [3]. Blockchain has firstly been introduced together with a cryptocurrency called 'Bitcoin' by Nakamoto in 2008 [1]. Nakamoto proposed a public ledger idea that uses a peer-to-peer (P2P) approach to maintain a distributed append-only database, and named it 'blockchain'. The first ever application of blockchain is Bitcoin. Unlike all existing currencies at that time, the issuing of Bitcoin and subsequent circulation do not require a government or central bank to regulate and monitor. All the trust to Bitcoin comes from its strong immutability and transparency [8].

Blockchain has experienced several evolutions in the past decade. Blockchain is mainly used in various cryptocurrencies during its early history. The first significant evolution of blockchain technology is the debut of Ethereum in 2014, where 
the distributed application and permission mechanism have been applied into blockchain [9]. However, the cryptocurrency is still a compulsory component of blockchain projects as it is the key to maintain trust and consensus for the distributed ledger. In 2017, the Linux foundation formally released its open source project called Hyperledger and advertised it as the third generation of blockchain [10]. From then on, the cryptocurrency ceases to be a native component and the concept of 'smart contract' becomes the core feature of blockchain projects [4]. Nowadays, a blockchain application could be discussed in 4 dimensions (1) a distributed ledger, (2) the cryptography behind, (3) the choice of consensus protocol and (4) the smart contracts [11].

In blockchain, all the data is inserted into and maintained by a distributed ledger in form of transactions [12]. The ledger is composited with a series of blocks where one or multiple transactions are enclosed in. Compared with traditional databases, blockchain provides an immutable distributed ledger where all the operations of data insertion could not be solely completed by the requesting user, therefore the people can trust that the ledger won't be messed or attacked [3].

Consensus is the key property of a blockchain system. As the peers within the blockchain network do not trust each other, the consensus protocol has to define the proper rule of peer review and verification to maximize the prevention against any rogue node or malicious user. Bitcoin uses a singlehandedly consensus called 'proof-of-work', which defines the only genuine version of the new block comes from the peer node with the highest computational power. The proof-of-work is a typical consensus protocol that makes selection based on one unique characteristic of peer nodes. There are some other popular consensus protocols such as proof-of-stake, proofof-activity, proof-of-elapsed time, practical byzantine fault tolerance (PBFT), etc [11], [13].

Cryptography is used in blockchain to guarantee the data integrity in the distributed ledger. Firstly, each transaction or block contains a digital signature. Every data entity in the ledger, regardless a transaction or a block, has a unique digital signature which could be identified to a specific user and be verified by everybody [12]. Moreover, the integrity of a block and all enclosed transactions are protected by a Merkle tree hash function. Any slight change in the data will result in a completely different hash value. As each block contains that hash value of its previous block, any data change made within a block will destroy all the subsequently connected blocks in the same chain. For those reasons, it makes attacker impossible to forge or tamper the data stored in the ledger [11].

Smart contract stands for the event-driven program executed at the moment a transaction is performed. A smart contract exists as a reusable code snippet in the blockchain system which could be automatically triggered to carry out one or multiple contractual clauses at some specific circumstances. In a blockchain system, it could be simple as digital signature validation or financial balance checking [14]. However, the complexity of smart contract grows rapidly with the evolution of blockchain application from cryptocurrency to a bigger context in industry and business.

\section{B. Impact of Blockchain in Industry}

Blockchain is a stunning solution to build trust in a trustless environment. In the world of blockchain, it is extremely difficult to corrupt or destroy any information even without a commonly trusted authority. Some studies indicate that proper usage of blockchain incurs lower human cost and higher security [2], [3]. The industry also holds a bullish view to blockchain since its invention in 2008 and expects it to solve more concrete problems in the future. The Gartner has nominated blockchain and distributed ledger as one of top ten strategic technology trends for consecutive two years since 2017 [15], [16]. Deliotte, one of the largest professional service providers in the world, evaluates blockchain as a "game changer" in accounting industry as it could lower the career barrier and bear a large part of jobs of accountants and auditors [17], [18]. Some business reviewers also believe that the blockchain will be revolutionary to supply chain management - the features of blockchain, such as transparency, security, traceability and trust-independence, are particularly aligned with the future demands of supply chain management [6].

\section{Supply Chain Management and Blockchain}

Industrial supply chain has never been placed in such complex geographical and cultural environment like today. The globalization opens up much opportunity for supply chain, but meanwhile, it has unearthed many challenges. Trust is one of the most critical factors for the business collaboration in supply chain management [19]. Despite managing relationship among different parties within a supply chain has been widely studied and discussed in past decades, most of studies could only apply their theories to the trust existing in a longterm relationship. The concern of trust is still challenging in nowadays industrial supply chain which is filled with quick changes and short life cycle production [20]. In addition, delivery performance is another challenge in the supply chain management. Because of the huge uncertainty about the intransit delivery status which makes the delivery performance difficult to be precisely measured, the nowadays supply chain management in global pipeline urges for better visibility [21]. Fortunately, the cutting edge information technologies are believed to boost companies management capabilities [22]. Allen Jacques, the Vice President of Pharma Supply Chain, proposes a set of suggestions to achieve more accurate delivery performance in 2016, which includes adoption of real-time calculation, setting up a responsive monitoring, and standardization of performance metrics across the supply chain [23].

There is no lack of pioneers who believe that blockchain technology could benefit the industrial supply chain management and so far have already implemented some real applications. IBM is one of the major players of blockchain application in supply chain management, who has established cooperation with the giant of global retails market Walmart as well as the leading container shipment service company Maersk [5], [24]. In particular, the introduction of blockchain 
in global shipping industry, which is jointly initiated by IBM and Maersk, has gain support from customs and port authorities of a wide range of countries including the United States, Netherlands, Singapore and China as they believe that innovation can greatly reduce bureaucracy and risk from cyberattack and thereby increase the reliability and efficiency [25].

\section{AsSessment MOdeL}

In this section, we propose an assessment model of estimated delivery performance in supply chain management. Our assessment model could change the way that an business organization only passively evaluate its delivery performance at post-delivery stage. By adopting our proposed model, an organization can evaluate its estimated performance since the beginning of supply chain contract and actively make corresponding operational decisions or adjustments. We present our deduction on the traditional delivery performance assessment formula, and extract two external factors that may cause potential influences to the estimation.

The most commonly used metric for delivery performance in supply chain management is called "OTIF" (On-Time InFull) or "DIFOT" (Delivery In-Full On-Time) ${ }^{1}$ [21], [26], [27]. It is a percentage value used for delivery key performance index (KPI) assessment of an organization. The formula of OTIF calculation could be presented as:

$$
\text { OTIF }=\frac{\text { number of deliveries OTIF }}{\text { total number of deliveries }} \times 100 \%
$$

By making use of Eq. (1), a business organization could easily calculate its delivery KPI. However, this calculation is a static assessment and it could only be performed during post-delivery period. Now with the introduction of blockchain technology, we propose an estimated OTIF evaluation which is derived from traditional OTIF formula and could be calculated in real-time manner during or even prior to the delivery.

Notation 1. We describe the OTIF as the capability of delivery and we notate the quantity of such capability of an organization $m$ as a function $\operatorname{cap}($ Delivery $m)$.

Notation 2. We also notate all the direct suppliers of $m$ as a finite set $\mathbb{S}$, the delivery service providers appointed by suppliers within $\mathbb{S}$ as a set $\mathbb{S D}$, the delivery service providers who are selected by $m$ to deliver its products to the customer, as a set $\mathbb{D}$.

Notation 3. Moreover, we use two functions, $p$ and $d$, to concisely represent a business entity's capability of production and delivery.

Thus we transform the estimated OTIF calculation for organization $m$ to the Eq. (2):

$$
\begin{aligned}
\mathrm{OTIF}_{\text {est }} & =\operatorname{cap}\left(\text { Delivery }_{m}\right) \\
& =p(\mathbb{S}) \times d(\mathbb{S D}) \times p(m) \times d(\mathbb{D})
\end{aligned}
$$

Assumption 1. As the estimated OTIF is designed to be a pre-production metric to evaluate the expected delivery

\footnotetext{
${ }^{1}$ Both "OTIF" and "DIFOT" shares the same calculation formula. For the purpose of consistency, in this paper we use the notation of "OTIF" only.
}

performance at the end stage, we assume the capability of production of organization $m$, together with the capability of post-production delivery capability of organizations within set $\mathbb{D}$, as constants and do not make influence to the preproduction estimated OTIF assessment.

Assumption 2. To make this assessment model simple to be presented, we assume the production capability of organizations in set $\mathbb{S}$ are independent to each other. Same assumption also applies to the delivery capability of delivery service providers in set $\mathbb{S D}$.

Assumption 3. In addition, we also assume the overall capability, of either production or delivery in supply chain management, of a group of organizations are evaluated in weighted sum manner. We use $w(i)$ to represent the weight function for organization $i$.

With the deduction in Eq. (2) and 3 assumptions depicted above, we can further transform and simplify the equation of estimated OTIF formula to:

$$
\begin{aligned}
\mathrm{OTIF}_{e s t} & =k \times p(\mathbb{S}) \times d(\mathbb{S D}) \\
& \propto p(\mathbb{S}) \times d(\mathbb{S D}) \\
& \propto \sum_{i \in \mathbb{S}}(p(i) w(i)) \times \sum_{j \in \mathbb{S} \mathbb{D}}(d(j) w(j))
\end{aligned}
$$

By obtaining the equation (3), we find the actual capability of delivery on-time in-full of an organization is not purely determined by its own production and customer delivery - it may also be affected by the production and delivery capabilities of other upstream participants within the same supply chain, such as suppliers and delivery service providers. The estimated OTIF value will be accordingly discounted in case of any failure of delivery in-full on-time happens to some of upstream suppliers or delivery service providers within the same supply chain. This estimated OTIF calculation lays theoretical foundation of our proposed blockchain system to make the real-time predictive delivery performance metric feasible for the actual supply chain management.

\section{A BLOCKCHAIN FRAMEWORK}

We propose a blockchain system and we call it "DelivChain". The DelivChain could provide necessary data input for the assessment model that we introduced in the previous section. Unlike the popular public blockchains like Bitcoin and Ethereum, DelivChain is designed as a consortium chain which only allows access and contribution from permissioned users. A DelivChain instance is usually set up for a specific contract that involves a group of organizations distributed into different stages in a supply chain, and led by the final manufacturing company that integrates all materials and semifinished products into the final products for the customers. The functionality of DelivChain is implemented to capture all the data related to the latest status or progress of production and delivery throughout the supply chain and finally transform those raw data into analytics and business intelligence. The layered architecture design of DelivChain, which is shown in Fig. 1, unveils how a blockchain system defines the data 


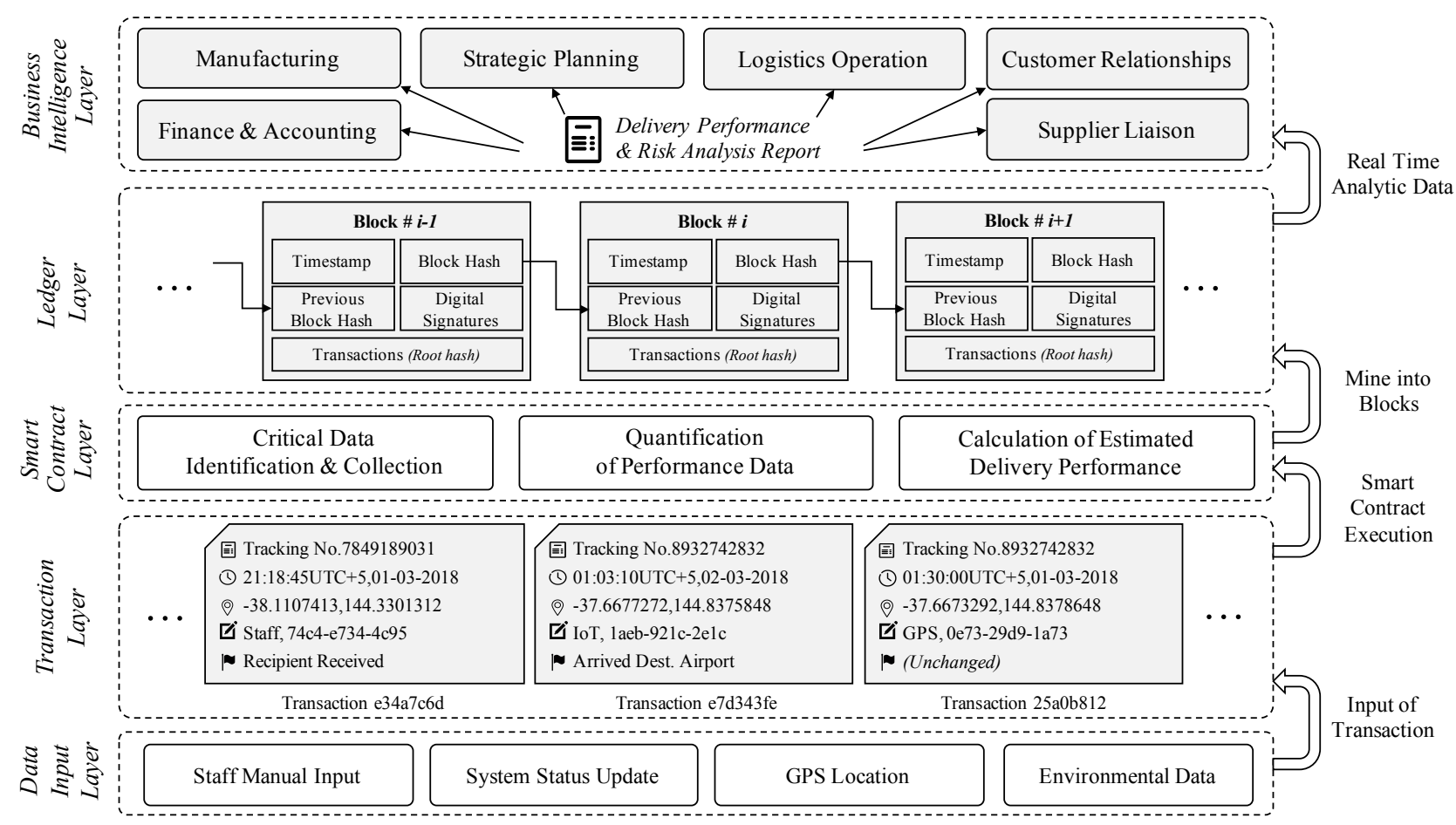

Fig. 1: A Layered Architecture of DelivChain System

model, collects the raw data, records into the immutable ledger and finally assists in achieving business intelligence by executing a well-designed smart contract. Next, we explain more details from prospectives of different layers.

Data Input Layer: The data input layer of DelivChain defines the source of raw input data that can be used in subsequent layers. The raw data comes from both production and delivery happening in the supply chain. It could be recorded manually by staff, or automatically by the system. Among them, the latter is usually generated either by the software logic periodically to reflect the latest progress, or by divers types of sensors used in productions and delivery. Thanks to emerging IoT devices and radio-frequency identification (RFID) technology, it makes real-time collection of geographic and environmental data possible in supply chain management. For example, having an awareness of the latest position of goods-on-delivery by tracking GPS data could help stakeholders of such delivery to understand the actual progress of the delivery service and thereby predict the expected time of delivery completion. Besides that, with the real-time environmental data of production or delivery in hands, such as temperature, water precipitation or other weather conditions a stakeholder can have a knowledge of any potential influence of environment to the expected outcome of production or delivery, for instance the traffic paralysis caused by flood or fog, power outrage caused by typhoon, etc. Once as many as possible raw data has been captured, our DelivChain system is able to move to the next layer to format those data and stored them in forms of transactions.

Transaction Layer: In this layer, all the real-time raw data captured in data input layer are formatted into a data structure called "transaction". A transaction is created immediately after a raw input data entity has been obtained, and then broadcast to the network for verification. A transaction instance contains one raw input data entity and other relevant details includes the ID of input staff or device, the tracking number, a timestamp and an optional note-to-user. All the transactions which have passed the verification are distributed in the network and sorted in chronological order by their timestamps. After that, those transactions will be encapsulated into a newly generated block at a future moment and then permanently stored into the blockchain ledger.

Smart Contract Layer: Smart contract is the core functionality of DelivChain to bring benefit to stakeholders of the supply chain. In DelivChain, a smart contract is defined as an event-driven program performed right after the submission of a new transaction. Our smart contract completes all rolebased logical operations to filter out useful raw data from the ledger and then transfer those real-time raw data to a series of values which are properly formated for business analytics. We summarize the functionality of DelivChain smart contract into 3 stages: (1) Critical data identification and collection. A smart contract deployed by an organization in the supply chain needs to keep tracking any change of the ledger and identify all the critical data it may concern. For example, the data implies production progress of its suppliers, or even suppliers' suppliers; and the data reflects the weather condition along the geographic route from supplier to the organization itself. (2) Quantification of performance data. With the data collected from the previous stage, smart contract in this stage needs to convert those data into quantitative values which could be used in benchmarking an organization's performance of production 


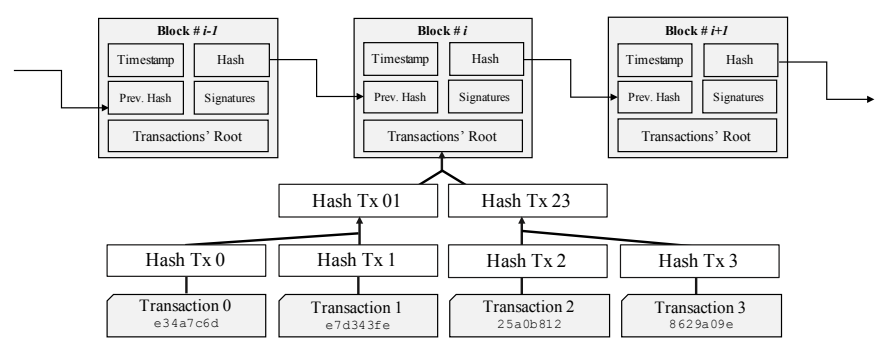

Fig. 2: Merkle Tree Hash Root Algorithm of Transactions in a Block

or delivery. For example, the data implies manufacturing delay of one supplier of the organization will be convert to a risk percentage value on the expected production capability of that supplier. An abnormal large water precipitation in the geographic region of a supplier will be evaluated and converted to another risk percentage value to the estimated performance of the delivery service provider. (3) Calculation of estimated delivery performance. In this stage, the smart contract refers to the assessment model that we have introduced in Section III, to put all the quantitative values obtained in the previous stages into consideration, and calculate the overall estimated delivery performance of the organization itself at that moment.

Ledger Layer: Once a transaction has been created and reached the threshold number of passed verification in the network, it becomes an "unsettled transaction". All unsettled transactions are placed in a distributed pool across the network and wait for next block miner, who is possibly a stakeholder of the supply chain, to package all of them into a new block and append into the ledger. A transaction is in a status called "settled" when it has been associated with a block on the ledger. One block has at least one transactions associated with it. As what Fig. 2 shows, those transactions associated to one block are not saved as plain text but a summary hash text generated by calculating the root of a Merkle Tree. Therefore a block, including all the transactions associated to it, are permanently immutable after being appended into the distributed ledger.

Business Intelligence Layer: The business intelligence layer denotes the application deployed upon our DelivChain system to perform business analytical tasks. It could be done purely automatically by using a business analytics software, or by mixture of systematic analytics and manual analysis. The goal of this layer is to transform the real-time quantitative values that reflect the estimated delivery performance to a periodical qualitative report written in business readable language and expression. This report is a key component to achieve business intelligence and extremely helpful when there is a big, unpredicted and unexpected impending hazard in the supply chain. With the report on hand, different departments are able to duly make corresponding decisions and well prepare for negative impact caused by the supply chain hazard.

\section{Discussion}

We introduce our assessment model in the Section III and then we present our blockchain framework DelivChain and
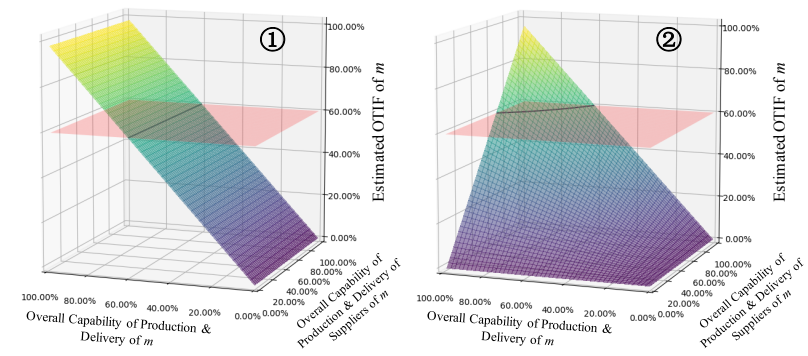

Fig. 3: The Comparison of Suppliers' Influence Between Traditional OTIF Assessment (Left) and Our Proposed Real-time Estimated OTIF (Right) with Expected Bottom Line (Alarm) Value Set to 60\%

explain how that blockchain framework realize our proposed assessment model in the supply chain management. In this section, we discuss the potential benefits that DelivChain can bring to the real business operation in supply chain.

\section{A. Trust and Transparency}

First, as a blockchain framework, our DelivChain platform enables all permissioned users to access and contribute to the distributed ledger. Owe to a group of ingenious cryptographic algorithms adopted in the blockchain technology, any data is impossible to be tampered or deleted once it has been appended to the ledger. Moreover, the distributed feature of the ledger of blockchain makes malicious user impossible to completely erase a part of ledger throughout the entire network. Compared with other traditional data storage solution, blockchain offers greater integrity, accountability, accessibility and non-repudiation to its users. In the use case of industrial supply chain, our DelivChain can serve as a trusted medium in a trustless environment that filled with omnipresent business competition. All participants in a supply chain can trust DelivChain platform even they do not trust each other. In addition, the DelivChain increases transparency within a supply chain since all users have to append the necessary data under promise into the ledger on time and without any reservation. Both trust and transparency are critical elements to build a healthy and sustainable business environment - our DelivChain could give a helping hand to it.

\section{B. Business Intelligence}

Data is the key component for a business organization to conduct strategy analytics and strategic analysis. Performing analytics and analysis based on historical data could help an organization in making not only accurate but also long-term decisions and thereby achieve business intelligence. Nowadays, the blockchain technology is the catalyst of the pursuit of business intelligence due to a series of advantages such as low cost, perfect security and high level automation. Blockchain moves a step further to provides business organization with real-time large-scale data. Take DelivChain as an example, the smart contract of DelivChain can filter out all irrelevant data from the ledger and perform quantitative analytics for users. By taking the capability of both suppliers and delivers into consideration, our proposed blockchain aided real-time metric 
called estimated OTIF could provide more comprehensive result compared with the traditional static evaluation. As shown in Fig. 3, the left diagram shows the traditional assessment that the delivery performance only depends on capability of the organization itself. After adopting our solution, which is shown in the right diagram, the organization can jointly oversee the self-capability, which could be summarized from historical data, as well as the real-time capability of suppliers. Even there is no decent in self-capability, negative impact from suppliers can still lower down the estimated delivery performance. Thus the organization can be alarmed and then make decision accordingly in case the predictive value falls below the expected bottom value.

\section{FUTURE WORK}

We make several assumptions in this paper and we exclude many factors from our scope. Those factors include but are not limited to economic factor, socio-cultural factor and political factors, which are existing in actual business study. Besides that, the metrics for delivery performance greatly vary in different organizations due to their diversity in management standards, organizational cultural and focus markets. For those reasons, our study still needs a lot of works in the future.

Firstly we plan to further our study in supply chain management to find a more mature and complete assessment model and enhance the functionality of DelivChain framework. Moreover, we are keen to explorer and study in more specific blockchain related issues which might be brought with the adoption of DelivChain, such as user privacy and access control management, data integrity at the input layer, infrastructure security and consensus policy. Last but not the least, we are open to explore more possible applications and consequent benefits that blockchain and relevant technologies could bring to nowadays industry, for example the intelligent accounting, risk analysis, inventory management, and etc.

\section{CONCLUSION}

In this paper, we conducted an interdisciplinary study on very concrete fields in both business and information technologies. We proposed an enhanced assessment model for realtime predictive delivery performance metric and we designed a blockchain framework called DelivChain to make our proposed assessment model possible in supply chain management. Furthermore, we discussed the potential benefits of DelivChain could bring to nowadays industrial supply chain from different perspectives. In the end, we shared our plan for the future work.

\section{REFERENCES}

[1] S. Nakamoto, "Bitcoin: A peer-to-peer electronic cash system," 2008.

[2] N. Levenson, "Trust In A Trustless System? How Ontology Could Bring Big Business To Blockchain," 2018, (Accessed 1-April-2018). [Online]. Available: https://hackernoon.com/trust-in-a-trustless-systemhow-ontology-could-bring-big-business-to-blockchain-fd73260ffee 9

[3] M. E. Peck, "Blockchain world-do you need a blockchain? this chart will tell you if the technology can solve your problem," IEEE Spectrum, vol. 54, no. 10, pp. 38-60, 2017.

[4] M. Swan, Blockchain: Blueprint for a new economy. "O'Reilly Media, Inc.", 2015.
[5] IBM, "Maersk and IBM to Form Joint Venture Applying Blockchain to Improve Global Trade and Digitize Supply Chains," 2018, (Accessed 1-April-2018). [Online]. Available: http://www-03.ibm.com/press/us/en/ pressrelease/53602.wss

[6] B. Dickson, "Blockchain has the potential to revolutionize the supply chain," 2016, (Accessed 1-April-2018). [Online]. Available: https://techcrunch.com/2016/11/24/blockchain-has-the-potentialto-revolutionize-the-supply-chain/

[7] S. Vachon and R. D. Klassen, "An exploratory investigation of the effects of supply chain complexity on delivery performance," IEEE Transactions on engineering management, vol. 49, no. 3, pp. 218-230, 2002.

[8] D. E. O'Leary, “Configuring blockchain architectures for transaction information in blockchain consortiums: The case of accounting and supply chain systems," Intelligent Systems in Accounting, Finance and Management, vol. 24, no. 4, pp. 138-147, 2017.

[9] V. Buterin, "Ethereum: Now Going Public," 2014, (Accessed 1April-2018). [Online]. Available: https://blog.ethereum.org/2014/01/23/ ethereum-now-going-public/

[10] Hyperledger, "Hyperledger Fabric 1.0 is Released!" 2017, (Accessed 1-April-2018). [Online]. Available: https://www.hyperledger.org/blog/ 2017/07/11/hyperledger-fabric-1-0-is-released

[11] D. T. T. Anh, M. Zhang, B. C. Ooi, and G. Chen, "Untangling blockchain: A data processing view of blockchain systems," IEEE Transactions on Knowledge and Data Engineering, 2018.

[12] S. Apte and N. Petrovsky, "Will blockchain technology revolutionize excipient supply chain management?" Journal of Excipients and Food Chemicals, vol. 7, no. 3, 2016.

[13] A. Castor, "A (Short) Guide to Blockchain Consensus Protocols," 2017, (Accessed 1-April-2018). [Online]. Available: https://www.coindesk. com/short-guide-blockchain-consensus-protocols/

[14] Bitcoinwiki, "Contract - Bitcoin Wiki," 2017, (Accessed 1-April-2018). [Online]. Available: https://en.bitcoin.it/wiki/Contract

[15] Gartner, "Gartner Identifies the Top 10 Strategic Technology Trends for 2017," 2016, (Accessed 1-April-2018). [Online]. Available: https://www.gartner.com/newsroom/id/3482617

[16] Gartner, "Gartner Identifies the Top 10 Strategic Technology Trends for 2018," 2017, (Accessed 1-April-2018). [Online]. Available: https://www.gartner.com/newsroom/id/3812063

[17] N. Andersen, "Blockchain technology - a game-changer in accounting?" 2016.

[18] S. Psaila, "Blockchain: A game changer for audit processes?" 2017.

[19] S. E. Fawcett, G. M. Magnan, and M. W. McCarter, "Benefits, barriers, and bridges to effective supply chain management," Supply Chain Management: An International Journal, vol. 13, no. 1, pp. 35-48, 2008.

[20] Q. Lu, M. Goh, and R. de Souza, "An empirical investigation of swift trust in humanitarian logistics operations," in The Palgrave Handbook of Humanitarian Logistics and Supply Chain Management. Springer, 2018, pp. 279-296.

[21] M. Christopher, Logistics \& supply chain management, 4th ed. Pearson UK, 2011.

[22] S. Lund, J. Manyika, and J. Bughin, "Globalization is becoming more about data and less about stuff," Harvard Business Review, 2016.

[23] A. Jacques, "5 Keys to Improving On Time In Full (OTIF) Deliveries," 2016, (Accessed 1-April-2018). [Online]. Available: http://fusionops. Inx.avisan.com/5-keys-to-improving-on-time-in-full-otif-deliveries/

[24] IBM, "Walmart, JD.com, IBM and Tsinghua University Launch a Blockchain Food Safety Alliance in China," 2017, (Accessed 1-April-2018). [Online]. Available: https://www-03.ibm.com/press/us/ en/pressrelease/53487.wss

[25] J. Gronholt-Pedersen, "Maersk, IBM to launch blockchain-based platform for global trade," 2018, (Accessed 1-April-2018). [Online] Available: https://www.reuters.com/article/us-maersk-blockchain$\mathrm{ibm} /$ maersk-ibm-to-launch-blockchain-based-platform-for-globaltrade-idUSKBN1F51DE

[26] H. Forslund and P. Jonsson, "Integrating the performance management process of on-time delivery with suppliers," International Journal of Logistics: Research and Applications, vol. 13, no. 3, pp. 225-241, 2010.

[27] M. Branch and V. P. B. Intelligence, "Performance benchmarking with big data," 2017. 